\section{A eficiência das emissões otoacústicas transientes e audiometria tonal na detecção de mudanças temporárias nos limiares auditivos após exposição a níveis elevados de pressão sonora}

\author{
Samanta Marissane da Silva Barros ${ }^{1}$, Silvana \\ Frota $^{2}$, Ciríaco Cristovão Tavares Atberino ${ }^{3}$, \\ Francisco Osterne ${ }^{4}$
}

\section{Resumo / Summary}

The efficiency of otoacoustic emissions and pure-tone audiometry in the detection of temporary auditory changes after exposure to high sound pressure levels

Palavras-chave: audiometria, emissões otoacústicas, perda auditiva temporária.

Keywords: audiometry, otoacoustic emissions, temporary hearing loss

\begin{abstract}
A
exposição ao ruído produz um efeito deletério sobre a saúde auditiva de trabalhadores. Objetivo: Realizar um estudo utilizando audiometria tonal e EOAT pré e pós-exposição a níveis elevados de pressão sonora, buscando informações para estabelecer a eficiência dos testes para detectar pequenas mudanças temporárias no limiar. Forma de Estudo: Coorte Transversal. Material e Método: A amostra compôs-se de trinta indivíduos otologicamente normais, com idade variando de 20 a 35 anos. Os testes audiometria tonal liminar e EOAT foram realizados em cabina acústica, pré e pós-exposição de 5 horas, a níveis elevados de pressão sonora (entre 80 a 90 dB). Resultados: Na audiometria tonal liminar as maiores mudanças dos limiares auditivos ocorreram nas freqüências altas (3000 a $8000 \mathrm{~Hz}$ ) pós-exposição a níveis elevados de pressão sonora. Os achados referentes às EOA revelaram que existe uma piora da reprodutibilidade nas freqüências de 1000 a $4000 \mathrm{~Hz}$ das EOAT, quando os trabalhadores foram expostos a níveis elevados de pressão sonora. Conclusão: Notamos que tanto a audiometria tonal liminar quanto às EOAT evidenciaram sensibilidade para detectar mudanças temporárias, estatisticamente significantes, nos limiares de audibilidade e reprodutibilidade, respectivamente, após a exposição a níveis elevados de pressão sonora.
\end{abstract}

\begin{abstract}
E xposure to noise has a harmful effect on the auditory health of workers. Aim: The main goal of this paper was to establish the role of pure-tone audiometry and evoked transient otoacoustic emissions in the detection of small temporary auditory changes after exposure to high sound pressure levels. Study Design: a cross-sectional cohort study. Material and Methods: 30 otologically normal subjects aged between 20 and 35 years were submitted to pure-tone audiometry and evoked transiente otoacoustic emissions before and after 5 hours of exposure to high sound pressure levels (betweeen 80 and $90 \mathrm{~dB}$ ). Results: For pure-tone audiometry the largest changes occurred at high frequencies - from $3 \mathrm{KHz}$ to $8 \mathrm{KHz}$ after exposure. The evoked transient otoacoustic emissions showed reduced reproductibility from $1 \mathrm{KHz}$ to $4 \mathrm{KHz}$ after exposure to noise. Conclusion: We noted that both puretone audiometry and evoked transient otoacoustic emissions had a role in detecting statistically significant changes in the auditory threshold and in reproductibility, after exposure to high sound pressure levels.
\end{abstract}

\footnotetext{
${ }^{1}$ Mestre em Fonoaudiologia pela Universidade Veiga de Almeida. ${ }^{2}$ Doutora em Disturbio da Comunicação Humana pela UNIFESP; Professora da UFRJ e UVA.

${ }^{3}$ Doutor em Otorrinolarigologosta pela USP; Professor da UERJ e UVA.

${ }^{4}$ Mestre em Distúrbios da Comunicação Humana pela UNIFESP; Professor da UVA. Universidade Veiga de Almeida.

Este artigo foi submetido no SGP (Sistema de Gestão de Publicações) da RBORL em 21 de maio de 2006. cod. 1958. Artigo aceito em 2 de julho de 2006.
} 


\section{INTRODUÇÃO}

Dentre os vários elementos de risco ocupacional, a exposição ao ruído pode ser indicada como um dos agentes que produz um maior efeito deletério sobre a saúde auditiva de indivíduos que trabalham em contato com esse elemento.

A exposição constante a níveis elevados de pressão sonora altera o bem-estar físico e mental do trabalhador, sendo o aparelho auditivo humano extremamente vulnerável à ação desse agente de risco ocupacional ${ }^{1}$.

Essa exposição pode desencadear alterações auditivas de graus variados, bem como problemas não-auditivos que vão se refletir sobre o comportamento social, familiar e laborativo do trabalhador.

A orelha interna, mais especificamente as células sensoriais da cóclea são muito sensíveis à exposição a níveis elevados de pressão sonora, sendo as células ciliadas externas as primeiras estruturas a serem afetadas.

As células ciliadas externas constituem o amplificador coclear, sendo estas importantes para a amplificação dos estímulos sonoros e determinadoras do funcionamento das células ciliadas internas, que são os verdadeiros receptores e codificadores cocleares ${ }^{2}$.

Níveis intensos de exposição ao ruído podem desencadear rupturas mecânicas da membrana basilar e das células sensoriais auditivas, e produzirem uma perda auditiva imediata de diferentes graus, os quais podem provocar uma alteração temporária ou permanente do limiar auditivo ${ }^{3}$.

Perdas auditivas por exposição ao ruído ocorrem, primeiramente, de forma reversível, através de mudanças temporárias do limiar auditivo na faixa de freqüencia de 2000 a $6000 \mathrm{~Hz}$. A presença de mudanças temporárias de limiar, em um maior ou menor grau, sinaliza um prognóstico de suscetibilidade para perdas auditivas neurossensoriais permanentes, fator este que vem sendo amplamente estudado pela comunidade científica ${ }^{4}$.

Mudança temporária de limiar auditivo pode ser definida como uma elevação do limiar de audibilidade que se recupera gradualmente após exposição ao ruído5.

Alguns autores ${ }^{6}$ descrevem a mudança temporária do limiar como um efeito de curto prazo que representa uma mudança da sensibilidade auditiva que retorna gradualmente ao normal, depois de cessada a exposição a níveis elevados de pressão sonora. As variações dos limiares estão diretamente relacionadas com a suscetibilidade individual, tempo de exposição ao ruído, intensidade e freqüência do som. As mudanças temporárias do limiar são mais freqüentes na faixa de 2000 e $6000 \mathrm{~Hz}$, principalmente se as variações dos limiares estiverem associadas à exposição a sons que excedem $80 \mathrm{~dB}$.

A exposição intensa ao ruído produz uma alteração na percepção sonora para as freqüências altas? ${ }^{7}$. Esse transtorno vem associado a uma dificuldade de detectar seletivamente a freqüência específica do sinal em ambiente ruidoso.

Estudo realizado sobre caracterização do sistema auditivo exposto ao ruído ${ }^{8}$ em 203 indivíduos evidenciou que $98 \%$ das audiometrias realizadas, após a exposição ao ruído, revelaram configurações alteradas. Os autores acreditam que existe uma etapa prévia às mudanças temporárias do limiar de audibilidade, visto que o sistema auditivo exposto ao ruído pode apresentar alterações auditivas subliminares, sem lesões aparentes.

O monitoramento auditivo dos trabalhadores, na maioria das indústrias, é feito através da avaliação audiométrica tonal liminar, instrumento este usado pela vigilância epidemiológica para a análise das alterações auditivas decorrente da exposição ao ruído?.

A audiometria tonal liminar se constitui em um exame subjetivo que é freqüentemente utilizado para avaliar a suscetibilidade acústica de um indivíduo que se expõe a agentes externos de risco auditivo, porém o mesmo sofre influências de diversos fatores, tanto de caráter funcional quanto psicossocial, podendo, dependendo do estado físico e emocional do trabalhador, gerar respostas e resultados auditivos irreais e contraditórios.

A avaliação audiométrica tonal possui alguns fatores não controláveis que podem interferir na execução, interpretação e resultado do exame audiométrico. A audiometria é um exame subjetivo e fatores inerentes ao indivíduo podem alterar o resultado da avaliação ${ }^{10}$.

Dessa forma, a avaliação auditiva ocupacional realizada somente com recurso da audiometria tonal liminar pode não retratar a real situação do funcionamento coclear do trabalhador, fato este que pode prejudicar todo trabalho de prevenção existente em uma indústria.

A aplicação de testes objetivos, como o das emissões otoacústicas evocadas, associado à avaliação auditiva ocupacional, seria um instrumento de grande valor para o monitoramento auditivo e análise da suscetibilidade para perdas auditivas de trabalhadores expostos a níveis elevados de pressão sonora.

A avaliação das emissões otoacústicas evocadas possibilita uma pesquisa direta do mecanismo de amplificação coclear das células ciliadas externas, pois se caracteriza como um exame objetivo, não-invasivo, sensível ao estado coclear, o que permite um monitoramento auditivo mais preventivo e efetivo ${ }^{11}$.

Kemp ${ }^{11}$ descreveu, em 1978, que a cóclea era capaz de produzir e receber sons. Essa capacidade coclear de gerar sons foi denominada de emissões otoacústicas. Kemp observou que esta energia (EOA) poderia ser mensurada no meato acústico externo de forma pouco invasiva, sendo que a presença de emissões otoacústicas seria indicativo de função coclear normal.

As emissões otoacústicas foram divididas em es- 
pontâneas e evocadas. As emissões otoacústicas espontâneas foram definidas por Kemp em $1989^{11}$ como sinais estacionários que podem ser registrados sem necessidade de estímulo acústico, estando presente em 50\% dos ouvintes normais. Já as emissões otoacústicas evocadas seria respostas cocleares das células ciliadas externas geradas por diferentes estímulos sonoros, sendo estas classificadas como: transiente, produto de distorção e estímulo freqüência.

A presença das emissões otoacústicas está relacionada a um funcionamento normal da cóclea e orelha média. Sua presença significa que a orelha média e a cóclea estão respondendo normalmente à estimulação sonora. Por meio desta, torna-se possível avaliar a sensibilidade do sistema auditivo eferente frente à exposição a níveis elevados de pressão sonora e drogas ototóxicas. Quando é descartada qualquer possibilidade de problemas estruturais de orelha média, a ausência de emissões otoacústicas pode estar diretamente associada a alterações cocleares de graus variados ${ }^{12}$.

Estudo sobre emissões otoacústicas evocadas ${ }^{13}$ realizado em 25 indivíduos, do sexo masculino e feminino, expostos a $100 \mathrm{~dB}$ NA durante 10 minutos revelaram que as emissões otoacústicas evocadas são capazes de detectar mudanças temporárias do limiar auditivo provocados por exposição ao ruído.

A inclusão da pesquisa das emissões otoacústicas na avaliação audiológica ocupacional de rotina propicia maiores conhecimentos sobre fisiopatologia da orelha interna ${ }^{14}$. O uso das emissões otoacústicas evocadas pode ser de grande utilidade na avaliação da susceptibilidade individual ao ruído, visto que alterações nas emissões podem ser detectadas antes que ocorram mudanças nos limiares auditivos tonais.

Buscando estabelecer a utilidade das emissões otoacústicas evocadas transientes para o monitoramento auditivo, Hotz et al. ${ }^{15}$ pesquisaram o efeito do ruído em 117 recrutas e 30 cadetes. Todos os militares se submeteram a uma avaliação audiométrica tonal liminar e pesquisa das emissões otoacústicas evocadas transientes, antes e depois de um período de 17 semanas, em que os mesmos eram expostos a níveis elevados de pressão sonora, devido a treinamento com armas de fogo. Uma análise comparativa, entre audiometria tonal liminar e EOAT, permitiu concluir que as EOAT são mais sensíveis para detectar alterações iniciais na cóclea, decorrentes da exposição ao ruído.

Os autores evidenciaram que a avaliação das emissões otoacústicas evocadas é um instrumento clínico muito efetivo para o monitoramento da cóclea, pois oferece múltiplas vantagens como objetividade e rapidez, além de grande sensibilidade e especificidade, o que permite a detecção de alterações cocleares sutis antes que sejam observadas na avaliação audiométrica tonal liminar.

Sendo assim, o uso das emissões otoacústicas evo- cadas, na avaliação auditiva ocupacional, seria de grande valia no monitoramento da audição de grandes grupos de indivíduos expostos a níveis elevados de pressão sonora.

A realização dessa pesquisa teve como objetivo estudar a avaliação audiométrica tonal liminar e emissões otoacústicas transiente, pré e pós-exposição a níveis elevados de pressão sonora, buscando informações para estabelecer a eficiência de ambos os testes para detectar pequenas mudanças temporárias no limiar auditivo.

\section{MATERIAL E MÉTODO}

Esta pesquisa foi analisada e aprovada pelo comitê de ética e pesquisa da Universidade Veiga de Almeida sob o número 28/04.

Participaram do estudo 30 pessoas otologicamente normais, sem comprometimento de orelha externa e/ou média, sendo todos do sexo masculino com idade variando de 20 a 35 anos e vínculo empregatício na tecelagem, de no mínimo 1 ano e máximo 3 anos, não tendo nenhum participante desempenhado função de risco auditivo, previamente, em outra empresa.

Todos os funcionários selecionados usavam equipamento de proteção individual do tipo "concha" ou "plugue" e desempenhavam atividades laborativas somente relacionadas a setores com níveis elevados de pressão sonora que alternava entre 80 a 90 dB NPS.

A primeira etapa de estudo constou da seleção da amostra para a pesquisa.

Para a seleção da amostra foi realizada: anamnese otológica, audiometria tonal liminar e timpanometria. O registro dos limiares auditivos foi obtido dentro de cabina acústica, cuja relação de níveis de pressão sonora e ruído ambiental, encontravam-se apropriados para cada freqüência pesquisada segundo padrão ANSI S.31 (1991).

A audiometria tonal liminar foi realizada com audiômetro Ad 27 Audiotest Interacústico, calibrado segundo padrão ANSI S.31 (1991) e fone TDH 39 P. A determinação dos limiares tonais foi realizada nas freqüências de: 250 , 500, 1000, 2000, 3000, 4000, 6000 e $8000 \mathrm{~Hz}$, através da técnica ascendente e descendente com estímulo tom puro, de forma contínua.

Todos os funcionários foram submetidos a uma avaliação timpanométrica com intuito de excluir problemas de orelha média. Na pesquisa timpanométrica foi utilizado o analisador de orelha média Amplaid 775, calibrado segundo padrão ANSI S.31 (1991).

Foram excluídos da amostra todos os funcionários cujos limiares audiométricos, na pesquisa pré-exposição a níveis elevados de pressão sonora, excederam $25 \mathrm{~dB}$ NA (ISO-1989) em uma ou mais freqüências testadas na via aérea, bem como todos os indivíduos portadores de curvas timpanométricas: As, Ad, B e C.

Os exames audiométricos que revelaram limiares 
auditivos normais e curva timpanométrica tipo A constituíram o grupo pré-exposição a níveis elevados de pressão sonora. Esses indivíduos foram encaminhados para a segunda etapa da pesquisa.

A segunda etapa da pesquisa consistiu na pesquisa audiométrica tonal liminar e registro das emissões otoacústica evocadas transiente pré e pós-exposição a 5 horas de níveis elevados de pressão sonora (80-90 dB). O estudo obedeceu à seguinte ordem:

Pré-Exposição a Níveis Elevados de Pressão Sonora

-Audiometria Tonal Liminar, sem que o funcionamento tivesse sido exposto a níveis elevados de pressão sonora. Repouso auditivo de 14 horas. (Já realizado na seleção da amostra)

-Medidas das Emissões Otoacústicas Evocadas Transientes, sem que o funcionário tivesse sido exposto a níveis elevados de pressão sonora.

Pós-Exposição a Níveis Elevados de Pressão Sonora

-Audiometria tonal Liminar, após 5 horas de exposição a níveis elevados de pressão sonora (80 a 90 dB).

-Medidas das Emissões Otoacústicas Evocadas transientes, após 5 horas de exposição a níveis elevados de pressão sonora (80 a 90 dB).

O monitoramento da função coclear consistiu no registro das emissões otoacústicas evocadas transientes, sendo utilizado o otoemissor Capella/Madsen acoplado a um computador Compaq/Presario.

O registro das emissões otoacústicas transientes foi feito dentro de cabina acústica. A análise das EOAT foi realizada na banda de freqüência de 1,2,3 e $4 \mathrm{KHz}$ com estímulo não-linear, clique de 70 dB NA e estabilidade de sonda igual ou maior que $70 \%$.

Na pesquisa das EOAT foi estudada a reprodutibilidade pré e pós-exposição a 5 horas de exposição a níveis elevados de pressão sonora. O critério para a avaliação de presença de resposta foi reprodutibilidade maior ou igual a 70\% em três freqüências (GATANU 1998).

Para todos os dados coletados foram calculadas as medidas de tendência central: média, mediana e desvio padrão. Os valores dos limiares de audibilidade e reprodutibilidade das EOAT pré e pós-exposição a níveis elevados de pressão sonora nas orelhas direita e esquerda foram comparados pelo teste t-student ( $\mathrm{t}$-crítico maior ou igual a 1,7) para verificar se houve diferenças entre dois grupos, antes e depois da exposição a níveis elevados de pressão sonora.

\section{RESULTADOS}

\section{Investigação da Audiometria Tonal Liminar}

Na pesquisa das medidas de tendência central (mé- dia) dos limiares de audibilidade encontramos mudanças (piora dos resultados) em todas as freqüências, após a exposição a níveis elevados de pressão sonora, em ambas as orelhas. As maiores mudanças de piora foram observadas nas freqüências de $8000 \mathrm{~Hz}$ na orelha direita e 6000 $\mathrm{Hz}$ na orelha esquerda.

As maiores mudanças nos limiares de audibilidade (piora) foram em $4000 \mathrm{~Hz}$ (76\% dos indivíduos) na orelha direita e $3000 \mathrm{~Hz}$ (90\% dos indivíduos) na orelha esquerda. A piora dos limiares de audibilidade variou de 5 a $20 \mathrm{~dB}$ na orelha direita e 5 a $15 \mathrm{~dB}$ na orelha esquerda (Gráfico 1 e 2 ).

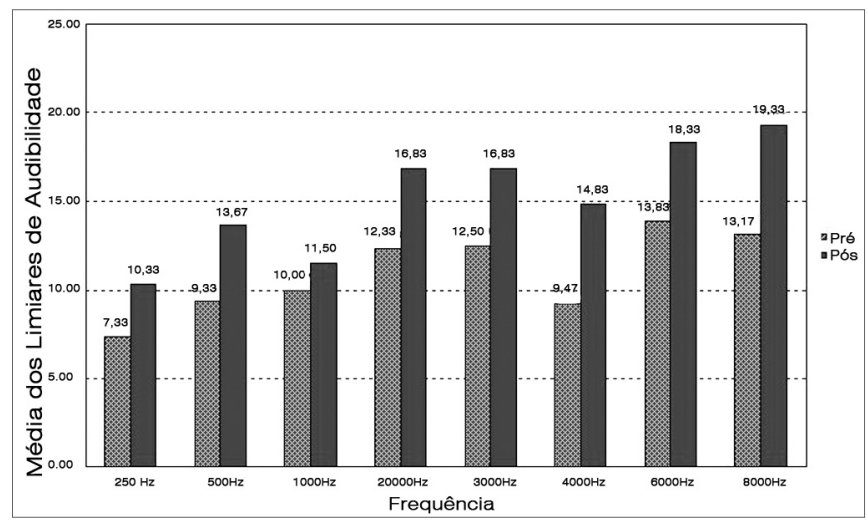

Gráfico 1. Média dos Limiares de audibilidade pré e pós exposição à níveis elevados de pressão sonora no sexo masculino na orelha direita.

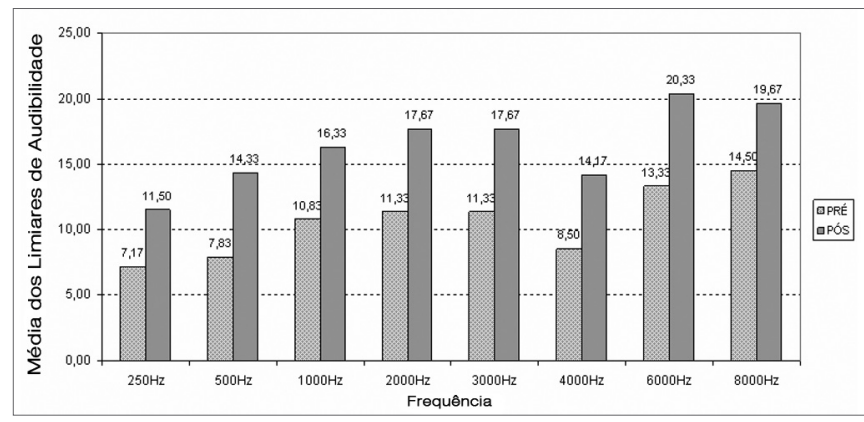

Gráfico 2. Média dos Limiares de audibilidade e medidas de tendências central, média, desvio padrão e mediana pré e pós-exposição ao ruído no sexo masculino na orelha esquerda.

No estudo comparativo entre os limiares de audibilidade obtidos pré e pós-exposição a níveis elevados de pressão sonora, por meio de teste paramétrico t-student, encontramos diferenças estatisticamente significantes ( $t$ crítico maior que 1,7$)$ em todas as freqüências em ambas as orelhas (Gráfico 3 e 4).

Investigação das Emissões Otoacústicas Evocadas Transientes

A análise dos resultados das emissões otoacústicas evocadas transientes revelaram diminuição da reprodutibilidade em todas as freqüências pesquisadas na orelha 




Gráfico 3. Valores do T -crítico - 1,7, nível de significância dos limiares de audibilidade em orelha direita.



Gráfico 4. Valores do T -crítico - 1,7, nível de significância dos limiares de audibilidade em orelha esquerda.

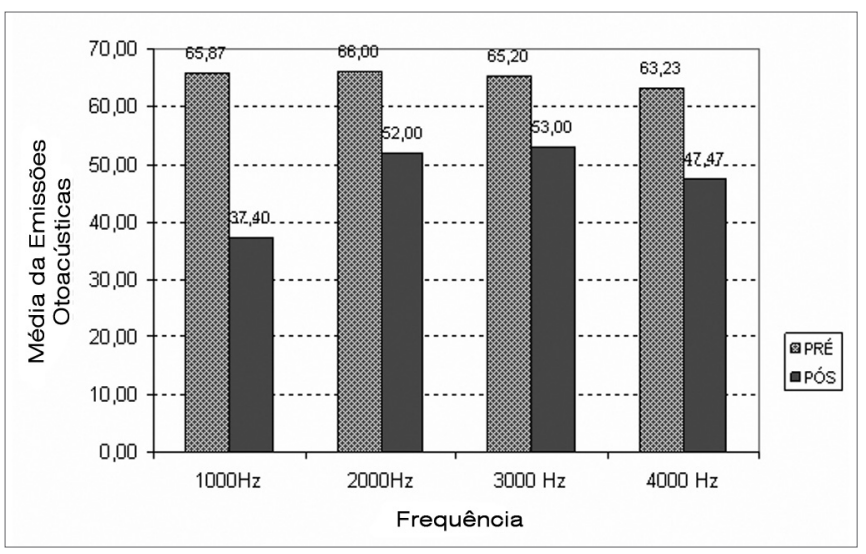

Gráfico 5. Média de reprodutibilidade das emissões otoacústicas evocadas transientes pré e pós exposição à níveis elevados de pressão sonora no sexo masculino na orelha direita



Gráfico 6. Média de reprodutibilidade das emissões otoacústicas evocadas transientes pré e pós exposição à níveis elevados de pressão sonora no sexo masculino na orelha esquerda. direita e esquerda pós-exposição a níveis elevados de pressão sonora (Gráfico 5 e 6).

Avaliando as medidas de tendência central (médias) de reprodutibilidade das EOAT pré e pós-exposição a níveis elevados de pressão sonora revelaram mudanças (piora) nas freqüências de 1000, 2000, 3000 e $4000 \mathrm{~Hz}$ na orelha esquerda e direita. As maiores mudanças de reprodutibilidade em relação aos valores médios foram observadas na freqüência de $1000 \mathrm{~Hz}$ na orelha direita e esquerda.

Neste estudo verificamos diminuição significativa (t-crítico maior que 1,7 ) de reprodutibilidade em todas as freqüências analisadas (Gráfico 7 e 8).



Gráfico 7. Valores do T -crítico - 1,7, nível de significância da reprodutibilidade das emissões otoacústicas evocadas transiente pré e pós-exposição à níveis elevados de pressão sonora no sexo masculino em orelha direita e orelha esquerda.



Gráfico 8. Valores do T -crítico - 1,7, nível de significância da reprodutibilidade das emissões otoacústicas evocadas transiente pré e pós-exposição à níveis elevados de pressão sonora no sexo masculino em orelha esquerda e orelha esquerda. 


\section{DISCUSSÃO}

Os valores encontrados na análise individual do limiar de audibilidade pré e pós-exposição de 5 horas a níveis elevados de pressão sonora revelaram que existe uma conformidade com a literatura ${ }^{4,6,7,8,16,17}$, no que se refere a mudança na configuração audiométrica e no limiar de audibilidade após exposição a níveis elevados de pressão sonora, bem como a grande incidência de alterações mais significativas nas freqüências alta. Estudos realizados em músicos expostos a ruído ${ }^{16,17}$ evidenciaram mudanças dos limiares auditivos após exposição a níveis elevados de pressão sonora. Todos os participantes apresentaram variação do limiar de audibilidade, principalmente nas freqüências de 3000, 4000 e 6000 Hz.

Outro estudo ${ }^{8}$ realizado com 203 indivíduos expostos ao ruído revelaram mudanças na configuração audiométrica em 98\% dos casos. Os achados sinalizam que existe uma etapa prévia às mudanças temporária do limiar de audição, visto que o sistema auditivo exposto ao ruído pode apresentar alterações auditivas subliminares, sem lesões aparentes.

Esses estudos vêm a corroborar com os achados obtidos na análise individual dos limiares de audibilidade da presente pesquisa, que revelaram maiores mudanças dos limiares auditivos nas freqüências altas, sendo as maiores mudanças observadas em $4000 \mathrm{~Hz}$ na orelha direita e 3000 Hz na orelha esquerda.

Os achados de nossa pesquisa reforçam as conclusões de outros autores $4,6,7,8,16,17$ de que existe uma correlação entre exposição a níveis elevados de pressão sonora e mudanças temporárias de limiar auditivo, sendo tais alterações mais evidentes nas freqüências altas (3000 a $8000 \mathrm{~Hz}$ ).

Em relação às emissões otoacústicas evocadas transientes, inúmeros estudos ${ }^{11-15}$ comentam a importância do uso das EOAT na detecção de alterações cocleares sutis, bem como no monitoramento auditivo de indivíduos expostos a níveis elevados de pressão sonora.

Ao compararmos nosso estudo com outros autores ${ }^{13,14}$ que estudaram as EOAT como recurso clínico utilizado para a constatação de mudanças temporárias de limiar auditivo e alterações cocleares, encontramos concordância no que diz respeito à diminuição da reprodutibilidade coclear para as freqüências médias e agudas.

Pesquisa realizada em 25 indivíduos, por meio do registro das emissões otoacústicas evocadas e audiometria tonal liminar, pré e pós-exposição a 100 dB NA durante 10 minutos revelou que a avaliação das emissões otoacústicas permite detectar mudanças temporárias do limiar auditivo. Em relação aos achados obtidos com a análise das EOAT, houve maior ocorrência de piora na amplitude média de resposta pós-estimulação para as freqüências agudas. O estudo também revelou que as EOAT são mais sensíveis à exposição ao ruído.

Estudo realizado em militares ${ }^{15}$ utilizando as emissões otoacústicas evocadas transiente revelou que a EOAT é um instrumento clínico que permite a detecção de alterações cocleares sutis, antes de serem revelada pela audiometria tonal liminar. Os autores encontraram alterações nas amplitudes das EOAT entre 2000 e 4000 Hz e nenhuma mudança nas freqüências de 250 e 500 HZ.

Pesquisadores ${ }^{15,13}$ afirmam que as EOAT permitem a detecção precoce de alterações cocleares sutis antes que sejam observados na avaliação audiométrica tonal liminar, sinalizando que as EOAT são mais sensíveis à exposição ao ruído, sendo capazes de detectar mudanças temporárias do limiar auditivo frente à exposição a níveis elevados de pressão sonora.

Esses achados, que comprovam que as EOAT permitem detectar mudanças temporárias do limiar auditivo, são compatíveis com os resultados da presente pesquisa, onde foram evidenciadas alterações (diminuição da reprodutibilidade) nas freqüências de 1000 a 4000 Hz que demonstram que as EOAT são sensíveis à detecção de mudanças temporárias de limiar auditivo, alterações cocleares, provocadas pela exposição a níveis elevados de pressão sonora, e que esta exposição promove uma piora na reprodutibilidade nas freqüências médias e altas.

\section{CONCLUSÃO}

Concluímos que ambos os testes, audiometria Tonal Liminar e Emissões Otoacústicas Evocadas Transiente demonstraram eficiência para detectar pequenas mudanças temporárias, estatisticamente significantes, nos limiares de audibilidade após exposição a níveis elevados de pressão sonora nas orelhas esquerda e direita.

\section{REFERÊNCIAS BIBLIOGRÁFICAS}

1.Nudelmann AA, Costa EA, Seligman E, Ibanez RN. Perda Auditiva Induzida por Ruído 2를. Rio de Janeiro: Revinter; 2001. p.79-84.

2.Oliveira JAA. O Mecanismo Eletrobiomecânico Ativo da Cóclea. Rev Bras Otorrinolaringol 1993;59(4):236-48.

3.Alberti PWRM. Deficiência Auditiva Induzida pelo Ruído. In: Filho OL Carlos R. Tratado de Otorrinolaringologia. 1르. São Paulo: Roca; 1994. p. 934-949.

4.Frota S, Iório MCM. Emissões Otoacústicas por Produto de Distorção e Audiometria Tonal Limiar: Estudo da Mudança Temporária do Limiar. Rev Bras de Otorrinolaringol 2002;68(1):15-20.

5.Melnick W. Saúde Auditiva do Trabalhador. In: KATZ J. Tratado de Audiologia Clínica 4⿳亠丷a Ed. São Paulo: Manole; 1999. p. 529-47.

6.Azevedo AP, Okamoto VA, Bernardi RC. Considerações sobre Ruído. In: Costa DF, Carmo JC, Settimi MM, Santos UP. Programa de Saúde dos Trabalhadores. São Paulo: Hucitec; 1989.

7.Prasher D. New Strategies for Prevention and Treatment of Noise-Induce Hearing Loss. Lancet 1998;352:1240-1.

8.Bonaldi LV et al. Exposição ao Ruído: Aspectos Funcionais do Sistema Auditivo em Humanos e Morfológicos em Modelo Animal Experimental. Rev Bras de Otorrinolaringol 2001;67(1):16-21.

9.Fiorini AC. A Importância do Monitoramento Audiométrico no Programa de Conservação Auditiva. Acust Vibr 1994;13:95-102. 
10. Gattaz G, Ruggieri M, Bogar P. Estudo das Emissões Otoacústicas Evocadas em Adultos Jovens Audiologicamente Normais. Rev Bras Otorrinolaringol 1994;60(1):15-8

11. Kemp DT. Otoacustic Emission: Basic Facts and Applications. Audiology in Practice 1989;3:1-4 .

12. Probst R et al. Otoacustic Emission in Ears with Hearing Loss. Am J Otolaryngol 1987;8:73-81.

13. Garcia AP et al. Emissões Otoacústicas Evocadas Transientes e Produto de Distorção em Adultos Normais: Estudo do Desvio Transitório do Limiar de Audibilidade após Exposição ao Ruído. Rev Pró-Fono 1999;11(2):53-9.
14. Fabiani M. Evoked Otoacustic Emission in the Study of Adult Sensorineural Hearing Loss. Br J Audiol 1993;27:131-7.

15. Hotz MA, Probst R, Harris FP. Monitoring the Effects of Noise Exposure Using Transiently Evoked Otoacustic. Acta Otolaryngol 1993;(Stockh)113:478-82.

16. Russo ICP et al. Um Estudo Comparativo Sobre os Efeitos da Exposição à Música de Trio Elétrico. Rev Bras Otorrinolaringol 1995;61(6):477-84.

17. Miranda CR, Dias CR. Perda Auditiva Induzida pelo Ruído em Bandas e Trios Elétricos de Salvador Bahia. Rev Bras Otorrinolaringol 1999;64(5):495-504 